\title{
The Human-Body-in-Coordination as Perceptual Instrument
}

\author{
Steven J. Harrison ${ }^{*}$ and Tehran J. Davis ${ }^{*}$ \\ ${ }^{*}$ Center for Cognition, Action, \& Perception and University of Cincinnati, USA \\ Email: harri2sv@ucmail.uc.edu, davtj@mail.uc.edu
}

\begin{abstract}
Recent evidence suggests that the human body in locomotor coordination performs dual roles, acting to propel the body over the surface of support, and embodying haptic information arising from and specific to the movement of the body as a whole with respect to the substrate. Here we show that blindfolded human subjects, trained to crawl using gait patterns that differed in the spatio-temporal symmetries defined with respect to the arms and legs in coordination, perceived distance travelled quadrupedally. These results suggest that 1) the body in coordination gives rise to a haptic measure of how one is moving through the world relative to the substrate and 2) that the measure that results is specific to the softly assembled global organization of the locomotor action system.
\end{abstract}

\section{Introduction}

Non-visual information about movement of the body relative to the environment is available with respect to the surface of support and with respect to the general background of resistance to acceleration [1]. Whereas the inertial variant of movementspecific information is detected by the vestibular system, the substrate variant is detected by the haptic perceptual subsystem of dynamic touch [2].

Studies of simple homing tasks in human subjects tend to implicate the substratal variant in non-visual distance perception [2]. These studies reveal a haptic measure of distance travelled that is invariant over changes in step amplitude, step frequency, and speed [3][4]; and that is specific to the spatiotemporal symmetries of the bipedal gait pattern employed [4]. For example, walk and run (bipedal gaits with equivalent spatiotemporal symmetries) produce equivalent measures of distance travelled; skip and run (bipedal gaits with differing spatiotemporal symmetries) produce non-equivalent measures of distance travelled.
One interpretation of these results is that the measure of distance travelled derived from the body in coordination is specific to the symmetries that characterize the organization of the locomotor action system. To test this generalization the current study investigated distance perception by human adults trained to crawl using gait patterns that differed in spatiotemporal symmetries of the arms and legs in coordination. We hypothesized that reports of distances measured by the body in quadrupdal coordination would depend upon the whether the gait patterns of measure and report shared identical or non-identical quadrupedal spatiotemporal symmetries.

\section{Method}

There were 18 participants in total: 9 in each experiment. Each person was pre-screened for physical fitness and absence of recent injury. All were outfitted with hand pads and knee pads to reduce discomfort from crawling.

Prior to data collection, participants were trained to crawl while blindfolded using two crawling gaits (see Figure 1). The particular trained crawling patterns varied across experiment. During the training session participants repeatedly watched an experimenter demonstrating and practiced blindfolded. To eliminate the possibility that prior visual knowledge of the experimental area would influence perceptual reports, the training session took place in a separate room from the main experimental area.

Following successful completion of the training, participants were blindfolded and led to the experimental area containing a long $(25 \mathrm{~m})$ felt path. The path was comprised of two parallel rails $(0.75 \mathrm{~m}$ apart) affixed to the ground. Crawling participants were self guided along the path by brushed contact with either of the rails.

On each experimental trial participants crawled along the path from a starting location to three target

This is an Open Access article distributed under the terms of the Creative Commons Attribution-Noncommercial License 3.0, which permits unrestricted use, distribution, and reproduction in any noncommercial medium, provided the original work is properly cited. 
distances $(3.8 \mathrm{~m}, 7.6 \mathrm{~m}, 11.4 \mathrm{~m})$ using one of the two trained crawling gaits. They were then instructed to report the distance they had travelled away from the starting location by turning around and crawling until they felt that they had travelled an equal distance (taking them back to the starting location).

Participants walked to each distance four times in each outbound gait, for a total of 24 randomized trials. Participants remained blindfolded until the conclusion of the experiment.

$\begin{array}{lll}\text { a) } & \text { b) } & \text { c) } \\ \pi & \pi & \pi \\ \pi & \pi & \pi \\ \pi & n & \pi \\ \pi & \pi & \pi \\ \pi & \pi & \pi \\ \pi & \pi & \pi \\ \pi & \pi & \pi \\ \pi & \pi & \pi \\ \pi & \pi & n \\ \pi & \pi & \pi\end{array}$

Fig.1. Trained quadrupedal movement patterns a) Pace, b) Trot and c) Prance.

\section{Results}

\subsection{Experiment 1}

In Experiment 1 participant's measure of outbound distance $(\mathrm{M})$ was obtained by crawling out using either a normal trot or a prancing trot gait pattern. Participants report (R) of outbound distance travelled was produced using the normal trot.

For the trot pattern contra-lateral arms and legs moved together, and the movement of arms and legs were qualitatively similar (Figure $1 \mathrm{~b}$ ). The prancing trot was similar to the normal trot in that contralateral arms and legs moved in unison; however the movement of the arms was qualitatively different from the movement of the legs. Specifically, during the swing phase the arm was raised up and tucked into the chest before returning to the ground (Figure 1c). Of note, whereas the movement of the legs was similar in across the two gait patterns considered, the prancing trot is characterized by less quadrupedal spatiotemporal symmetry than the normal trot [5].
A difference in quadrupedal spatio-temporal symmetries across measure and report was found to result in differences at reports of distance travelled $F(1,8)=67.95, p<.001$, number of steps taken $F(1$, $8)=46.20, p<.001$, and the time taken to make the report $F(1,8)=11.23, p<.05$, with the measure gait prance tending to result in increasingly smaller reports of distance travelled $F(2,16)=269.77, p<$ .001 , number of steps taken $F(2,16)=224.13, p<$ .001 , and the time taken to make the report $F(2,16)=$ $6.07, p<.05$, with increasing outbound distance, than when trot was the measure gait (see Figure 2).
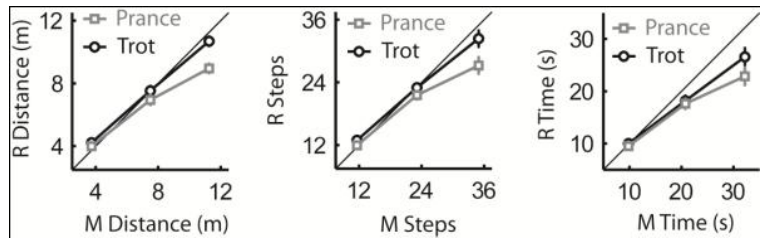

Fig.2. Experiment 1 results. Distances (left), steps (middle) and time taken (right) at report $(R)$ as a function of distance and gait at measure (M).

\subsection{Experiment 2}

For Experiment 2, participants walked outbound using either a normal trot (contra-lateral arms and legs moving in unison, Figure 1b) or a pace (lateral arms and legs moving in unison, Figure 1a). Both the normal trot and the pace patterns have equivalent spatiotemporal symmetry [5].

The absence of a difference in quadrupedal spatio-temporal symmetries across measure and report was found to result in no differences at reports of distance travelled, number of steps taken, or the time taken to make the report, all $F^{\prime}$ s $<1$ (see Figure $3)$.
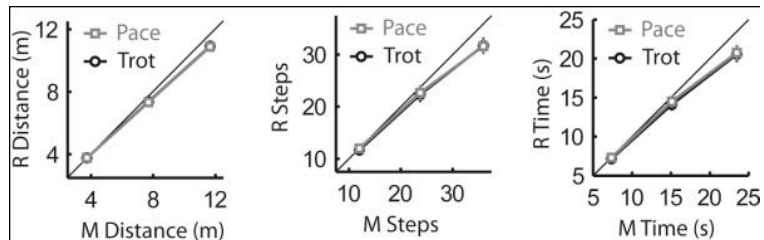

Fig.3. Experiment 2 results. Distances (left), steps (middle) and time taken (right) at report $(R)$ as a function of distance and gait at measure (M).

\section{Conclusion}

Consistent with previous studies of bipedal nonvisual distance perception [5], the present study replicates the basic earlier finding that the haptic 
perception of distance travelled is specific to the spatio-temporal symmetries of the movement pattern employed across measure and report. Reports of distances travelled were found to be equivalent in cases where gaits at measure and report shared the same spatio-temporal symmetries. In contrast, measure gaits that differed in spatio-temporal symmetries from report gaits resulted in distinct reports of distance traveled.

These results presented here also support the conjecture that gait symmetry offers a general principle for understanding how the movements of the body in locomotor coordination generates information specifying the bodies changing relationship to the substrate. Taken as a general principle, the gait symmetry perspective suggests that the measure of distance obtained by the body in coordination is not interpretable in terms of the movement of the individual limbs per-se but rather is specific to the global phase [6] of the action system assembled.

\section{References}

[1] Mittelstaedt, M. L. \& Mittelstaedt, H. (2001). Idiothetic navigation in humans: estimation of path length. Experimental Brain Research, 139, 318-332.

[2] Gibson, J. J. (1966) The senses considered as perceptual systems. Boston, MA: Houghton Mifflin.

[3] Schwartz, M. (1999) Haptic perception of the distance walked when blindfolded. Journal of Experimental Psychology: Human Perception and Performance, 25, 852-865.

[4] Turvey, M.T., Romaniak-Gross, C., Isenhower, R. W., Arzamarski, R., Harrison, S. J. \& Carello, C. (2009). Human odometer is gait-symmetry specific. Proceedings of the Royal Society B, 276, 4309-4314.

[5] Golubitsky, M., Stewart, I., Buono, P.-L. \& Collins, J. J. (1999). Symmetry in locomotor central pattern generators and animal gaits. Nature, 401, 693-695.

[6] Lamoth, C. J. C., Daffertshofer, A., Huys, R. \& Beek, P. J. (2009) Steady and transient coordination structures of walking and running. Human Movement Science, 28, 371-386. 